\title{
Precincts of Glomerular Disease Markers
}

\section{Pragasam Viswanathan*}

Renal Research Lab, School of Biosciences and Technology, Centre for Biomedical Research, VIT University, Vellore -632014, Tamil Nadu, India

\section{Editorial}

Several diseases affect the functionality of kidney, mainly by disturbing glomeruli, the tiny units within the kidney where blood is filtered. The glomerulus consists of a tuft of capillaries and associated cells that are responsible for filtration of small molecules while preventing the loss of larger molecules. The permeability barrier is damaged in glomerular diseases that lead to proteinuria. The damage on glomerulus is caused by variety of genetic and environmental factors.

In most of the occasions, renal biopsy is needed to make a definitive diagnosis of the cause of disease. The utility of renal biopsy is limited with several aspects. Because the biopsy contains only a fraction of kidney, in most of the cases it may not precisely represent the disease if the affected portion of the kidney is not sampled. In other cases, the disease may be so far advanced that diagnostic features are obscured. Urine testing for biomarkers could replace renal biopsy as a simple, safe, and accurate test that could be repeated to follow progression of the disease and monitor response to therapy.

Literature survey indicated that PubMed represents approximately 2000 independent research and review articles on glomerular injury markers. Similarly around 120 articles in clinical case reports related to glomerular diseases till December 2015. All these investigative and review articles represents some of the potential urine markers for diagnosis of glomerular disease, but none has been confirmed to differentiate between causes of glomerular diseases.

Many of the proposed markers are urinary cytokines, additionally, Levels of monocyte chemoattractant protein-1 (MCP-1), IL-6, vascular cellular adhesion molecule-1, the complement degradation product C3d, and urinary free light chains have also been proposed as markers of glomerular diseases. Further, Urinary concentrations of vascular endothelial growth factor, IL receptor 1 antagonist, IL-17, TNF- $\alpha$, Neutrophil Gelatinase Associated Lipocalin (NGAL), B-type Natriuretic peptide, Cystatin C and CD46 have been proposed as markers of specific glomerular diseases [1,2]. Urinary macrophages, podocytes, and associated proteins are also potential markers for glomerular diseases.

The presence of plasma proteins in urine of patients with any renal diseases provides an opportunity for discovery of new biomarkers. The glomerular filtration barrier has been the subject of major study for decades. Differences in the charge of several urinary macromolecules (Albumin, Heparin sulfate, Ferritin, horseradish peroxidase and globulins) are known to influence its permeability at the glomerulus. In any systemic or sclerotic renal disease, the glomerular permeability barrier is altered, resulting in proteinuria.

If changes in size and charge permeability occur independently and are specific to the disease, then these changes could be used to predict the cause of glomerular diseases. The studies conducted in both animal models and clinical investigations on diabetic nephropathy, glomerulonephritis, pre-eclampsia, and systemic lupus erythematosus (SLE) indicated that Nephrin could be early marker for glomerular injuries. Currently urine micro-albuminuria is used as an indicator of glomerular injury. But, both animal and human studies have demonstrated that nephrinuria occurs early in glomerular injury, preceding albuminuria, and that there is a positive correlation with severity of renal diseases [3].

The study conducted by Eriguchi et al. in both human and animal studies indicated that urinary angiotensinogen could be one of the markers for proteinuric nephropathies. Immuno-staining conducted by the same group revealed that rat AGT was detected in injured podocytes, and filtered human AGT was seen in superficial proximal tubules, but not in injured podocytes, suggesting AGT generation by injured podocytes [4].

Studies conducted from our laboratory on both animal model and clinical studies indicated that soluble cluster differentiation 36 (sCD36) could also be one of the plausible prognostic marker for diabetic nephropathy. Our study has reported the presence of sCD36 in urine for the first time. The level of sCD36 in both plasma and urine are found to be associated with the kidney markers such as urea, creatinine and albuminuria of diabetic subjects. The study clearly indicates that compared to control subjects, patients with T2DM have progressively increasing trend in both plasma and urine levels of sCD36 with increasing severity of nephropathy [5].

The major limitations in screening of all these disease-specific biomarkers in urine are complicated by significant changes in the proteome during the day. These changes are likely caused by variations in the diet, metabolic or catabolic processes, circadian rhythms, exercise, as well as circulatory levels of various hormones. The reproducibility of any analysis is reduced by these physiological changes, even if the analytical method shows high reproducibility. However, these variations appear mostly limited to a fraction of the urinary proteome; a large portion remains unaffected by these processes.

Because of these limitations in the urinary proteins detections, now most of the researchers are interested in understanding the pathogenesis of the renal diseases such as IgA nephropathy, membranous nephropathy and focal segmental glomerulosclerosis by systems biology approach. Advances in the molecular technology now allow genome wide high throughput characterization of genes and gene products from the urine samples. The Nephrotic Syndrome Study Network (NEPTUNE) study aims to integrate comprehensive clinical and pathologic phenotypes with extensive analysis of the molecular regulatory networks active in glomerular diseases. The NEPTUNE will prospectively procure human samples for analyses focused on genome-wide profiling of genetic variation, epigenetic markers and transcriptomic, proteomic,

*Corresponding author: Dr. Pragasam Viswanathan, Professor, Renal Research Lab, Bio Medical Research Centre, School of Bio Sciences and Technology, VIT University, Vellore-632 014, Tamil Nadu, India, Tel: +91-416-220 2583; E-mail: pragasam.v@vit.ac.in

Received December 17, 2015; Accepted December 22, 2015; Published December 30, 2015

Citation: Viswanathan P (2015) Precincts of Glomerular Disease Markers. J Kidney 1: e103. doi:10.4172/2472-1220.1000e103

Copyright: ( 2015 Viswanathan P. This is an open-access article distributed under the terms of the Creative Commons Attribution License, which permits unrestricted use, distribution, and reproduction in any medium, provided the original author and source are credited. 
and metabolomic profiles. Emerging systems biology approaches will be used to define associations between these multi-phasic and interrelated genome scale profiles and the detailed clinical and structural phenotypes. The NEPTUNE study will establish a core molecular data set containing genetic profiles and renal tissue, compartment-specific, genome wide mRNA expression data to facilitate multi- and transdisciplinary research exploration along the genotype - phenotype continuum [6].

Metabolomics appears to be a snapshot of the chemical fingerprints identifying specific cellular process. The metabolomics study carried by Gao et al. revealed a better understanding of the pathogenic profile of membranous nephropathy (MN). The study indicated that 26 urinary and 9 serum metabolites were screened using Gas-Chromatography and Mass Spectral analysis in low urinary protein $\mathrm{MN}$ and high urinary protein MN groups. These metabolites (sugar alcohols, dicarboxylic acid, hydroxylic acids and phenolic acid) are direct result of kidney damage [7]. Similarly, the study conducted by Won et al. on acute kidney injury indicated that early detection of decreased level of 3 -indoxylsulfate in the urine of human subjects and animal models, but at the same time its concentration is progressively increased in the serum of the same. For other glomerular diseases, new and innovative approaches are necessary to find other reliable and valid biomarkers for use in glomerular disease. Sophisticated techniques such as proteomics and metabolomics have become popularized in renal disease diagnosis for the patient management in the hospital sectors.

\section{References}

1. Fassett RG, Venuthurupalli SK, Gobe GC, Coombes JS, Cooper MA, et al (2011) Biomarkers in chronic kidney disease: a Review. Kidney Int 80: 806-821.

2. Won AJ, Kim S, Kim YG, Kim KB, Choi WS, et al. (2015) Discovery of urinary metabolomic biomarkers for early detection of acute kidney injury. Mol BioSyst 12: 133-144.

3. Kandasamy Y, Smith R, Lumbers ER, Rudd D (2014) Nephrin - a biomarker of early glomerular injury. Biomarker Research 2: 2-8.

4. Eriguchi M, Yotsueda R, Torisu K, Kawai Y, Hasegawa S, et al. (2015) Assessment of urinary angiotensinogen as a marker of podocyte injury in proteinuric nephropathies. Am J Physiol Renal Physiol, ajprenal 002602015.

5. Shiju TM, Mohan V, Balasubramanyam M, Viswanathan P (2015) Soluble CD36 in plasma and urine: a plausible prognostic marker for diabetic nephropathy. $J$ Diabetes Complications 29: 400-406.

6. Gadegbeku CA, Gipson DS, Holzman LB, Ojo AO, Song PX (2013) Design of the Nephrotic Syndrome Study Network (NEPTUNE) to evaluate primary glomerular nephropathy by a multidisciplinary approach. Kidney Int 83: 749756.

7. Gao X, Chen W, Li R, Wang M, Chen C, et al. (2012) Systematic variations associated with renal disease uncovered by parallel metabolomics of urine and serum. BMC Syst Biol 6: S14. 